\title{
Technokratie \\ oder Gemeinschaftswerk?
}

\author{
Expertengremien und Partizipation in der Energiewende
}

\begin{abstract}
Jörg Radtke, Seminar für Sozialwissenschaften, Universität Siegen, Adolf-Reichwein-Straße 2, 57068 Siegen (radtke@politikwissenschaft.uni-siegen.de) (1) https://orcid.org/0000-0002-6540-8096

Emily Drewing, Seminar für Sozialwissenschaften, Universität Siegen (emily.drewing@uni-siegen.de) (1) https://orcid.org/0000-0002-2170-9305
\end{abstract}

Maßgeblich beeinflusst durch Expertenkommissionen folgt die Energiewende bisher vornehmlich technisch-ökonomischen Logiken, während Laienperspektiven kaum Berücksichtigung finden. Dies kann zum Eindruck einer Technokratie beitragen und die gesellschaftliche Unterstützung für die Energiewende erodieren. So kommt der partizipativen Technikfolgenabschätzung besondere Bedeutung zu, um Ziele politischer Entscheiderinnen und Entscheider mit Interessen der Bürgerinnen und Bürger besser zusammenzubringen. Indes weist die partizipative TA in der Energiewende wesentliche Schwachpunkte auf. Der Beitrag umreißt die Rollen von Expertengremien und partizipativer TA in der Energiewende und diskutiert hieraus resultierende Probleme und Chancen einer stärker responsiven Governance.

\section{Technocracy or joint effort?}

Expert committees and participation in the German Energiewende

To date the energy transition has primarily followed technological and economic rationales and has been, significantly influenced by expert commissions, while laypersons' perspectives have hardly been taken into account. This can add to the impression of a technocracy and erode public support for the energy transition. Thus, participatory technology assessment is seen as crucial to align political decision makers' perspectives with those of citizens. However, participatory technology assessment has major shortcomings with regard to the energy transition. This article outlines the roles of expert committees and participatory technology assessment in the energy transition and discusses resulting problems and opportunities for more responsive governance.

Keywords: Energiewende; energy transition; technology assessment; technocracy; participation

This is an article distributed under the terms of the Creative Commons Attribution License CCBY 4.0 (https://creativecommons.org/licenses/by/4.0/)

https://doi.org/10.14512/tatup.29.3.36

Submitted: 15. 06.2020. Peer reviewed. Accepted: 21.10.2020

\section{Technikfolgenabschätzung und Energiewende}

2011 nahm mit dem kurz nach der Fukushima-Katastrophe beschlossenen Atomausstieg auch die Energiewende an Fahrt auf. Als soziotechnisches Großprojekt - es geht um nicht weniger als die Transformation aller Sektoren der Energieproduktion und -distribution sowie grundlegende Veränderungen beim Energiebedarf - stellt sie naturgemäß einen wesentlichen Gegenstand zeitgenössischer TA dar. Interessanterweise befasst sich das erst 1990 gegründete TA-Büro am Deutschen Bundestag zwar durchaus mit einschlägigen Untersuchungen (etwa im Themenfeld „Energie, Ressourcen, Umwelt“), Kernthemen der Energiewende bleiben jedoch weitgehend unbehelligt (TAB 2020). Aus der Perspektive der TA-Forschung kollidieren in der Energiewende zwei typische, indes sehr unterschiedliche Zugänge zum Themenfeld: das Vorsorgeprinzip (Gleich 2013) mit der Perspektive Betroffener von Energiewende-Maßnahmen (Grunwald 2010). Für die Bundes-, Landes- und Bezirksregierungen ist der Aspekt der Vorsorge im Sinne eines nachhaltigeren Energiesystems, das eine stabile Versorgung garantiert, vorrangig, während aus Sicht der Betroffenen Eingriffe in die vertraute Umgebung, Kosten sowie (mit Blick auf Anlagenbetreiber) Vergütungen und Sicherheiten im Vordergrund stehen.

Nach wie vor findet die Energiewende im Allgemeinen hohe Zustimmung in Politik und Gesellschaft (AEE 2020). Längst ist jedoch eine kritischere und reflexive Phase erreicht, in der die Öffentlichkeit mit differenzierterem Blick auf Fortschritte und Richtungsentscheidungen schaut (Setton 2019). Die Umsetzung der Energiewende ist politisch umkämpft und mit mannigfaltigen Unsicherheiten behaftet (Radtke 2020). Technische Fortschrittlichkeit und Verlässlichkeit sowie Wirtschaftlichkeit und Wettbewerbsfähigkeit markieren zentrale Anforderungen an das zukünftige Energiesystem. Im Policy-Vergleich ist die Energiewende-Politik denn auch durch zahlreiche beratende Gremien stark expertokratisch und auf Wissensebene konsultativ geprägt (Gründinger 2017). Im Jahr 2011 erklärte die von der Bundesregierung einberufene Ethik-Kommission die Energiewende zum 
Gemeinschaftswerk und bekräftigte darin den erforderlichen gesellschaftlichen Rückhalt, den es durch kollektive Beteiligung zu sichern gälte (Ethik-Kommission Sichere Energieversorgung 2011, S. 37 ff.). Nach wie vor braucht die Energiewende breite gesellschaftliche Unterstützung, so sie ihren (nicht unwesentlichen) Beitrag zur Reduktion der Treibhausgasemissionen leisten soll - man denke etwa nur an die Auswirkungen diverser Widerstände und Klageverfahren auf den Windenergieausbau. Es wird somit einerseits verstärkt auf Expertenkommissionen gesetzt, während andererseits verschiedene Modi der Bürgerbeteiligung und Mitbestimmung in Anschlag gebracht werden. Im Folgenden diskutieren wir den häufigen Einsatz von Expertengremien, Anspruch und Wirklichkeit der Partizipation in der Energiewende sowie mögliche Zukunftsperspektiven.

\section{Technokratie der Expertenkommissionen?}

Die Idee der Konsultation basiert im nationalstaatlichen Modell verfasster Demokratien darauf, dass Gremien oder Institutionen im Spannungsverhältnis zwischen repräsentativen und partizipativen Formen der Demokratie möglichst objektiv, ausgewogen und fachlich fundiert vermitteln (Neunecker 2016). Im Kontext der Energiewende finden Konsultationen in Form beratender (Experten-)Gremien der Parlamente und Regierungen sowie offenerer, stärker partizipativer und bürgerorientierter (Laien-) Konsultationen statt, welche zumeist durch spezielle Institutionen (z. B. Bundesnetzagentur) und auf regionaler Ebene (z. B. bei der Entwicklung von Energiekonzepten) eingesetzt werden. Je höher indes die politische Ebene, desto weniger finden sich diese Formate. Demnach lässt sich die Energiewende auf
Gesellschaft. Trotz dieser eher ökologisch geprägten Wurzeln wird die Energiewende heute stark durch ökonomisch-technische Relevanzsetzungen geprägt (Haas 2017). Dies resultiert aus dem Zusammenwirken diverser, teils anlassbezogener, teils kontinuierlich bestehender Expertengremien (welche Parlament und Regierung beraten) ${ }^{1}$. So hat sich ein selbstständiges ExpertenRegime ausgeprägt, das durch komplexe und verschachtelte Akteurskonstellationen (inkl. diverser Advocacy Coalitions und Vetoplayer) geprägt ist.

Die Idee der Technokratie beschreibt im Kern eine Dominanz von Expertenmeinungen sowie rationaler Logiken und formalisierter Strukturen bei (politischen) Entscheidungsfindungsprozessen, die freilich im Ergebnis keineswegs zu unumstrittenen Entscheidungen führen (Habermas 2013). In der TA werden die typischen Probleme des durch Expertengremien begründeten Wissensregimes umfassend beschrieben: Vorschläge zur Gestaltung, Prognosen und evolutorische Dynamiken führen aufgrund von Komplexitäten, Unsicherheiten und Selektivität sowie hinter vermeintlicher Objektivität wirkenden normativen Paradigmen zum „Expertendilemma“ (Grunwald 2010, S. 154). Auf Gutachten folgen Gegengutachten, der öffentliche Eindruck von Einheit und konsensualem Handlungswillen wird durch Zersplitterung und Fraktionsbildung unterminiert. Zudem bleibt vieles vage, woraus im politischen Tagesgeschäft ein gewisser Pragmatismus folgen kann (Rothe 2017) - bzw. ein MuddlingThrough, wie Czada (2019) der Bundesregierung mit Blick auf die Energiewende attestiert. Ergebnis dieser expertokratisch geprägten, dabei im Gesamteindruck eher passiven, ungerichteten und wenig ambitionierten Energiepolitik sind diverse Parallelstrukturen, welche die Koordination der Multilevel Governance und länderübergreifende Konsensbildung erschweren (Chemnitz 2019; Radtke 2018).

\section{Kommissionen stehen grundsätzlich unter dem Verdacht, expertokratische Entwicklungen zu fördern und Sachzwänge zu begründen.}

Bundesebene als ein stark durch Expertinnen und Experten geprägtes technisches Großvorhaben charakterisieren - und war es mit Blick auf die Besetzung der Ethik-Kommission Sichere Energieversorgung, die 2011 einen raschen Atomausstieg nahelegte und damit die Energiewende in Gang brachte, von Anfang an. Wesentliche Vorarbeiten hatten zuvor bereits der Rat für Nachhaltige Entwicklungen mit der Deutschen Nachhaltigkeitsstrategie (Die Bundesregierung 2002) sowie der Sachverständigenrat für Umweltfragen, der 2010 eine Stellungnahme zur Vision einer vollends erneuerbaren Stromversorgung bis 2050 abgab (SRU 2011), geleistet. Auch der Wissenschaftliche Beirat der Bundesregierung Globale Umweltveränderungen (WBGU) arbeitet seit 1992 an Entwürfen für eine nachhaltige
Kommissionen stehen grundsätzlich unter dem Verdacht, expertokratische Entwicklungen zu fördern und Sachzwänge zu begründen (Haring 2010). Häufig nur als Mittel zur Bestätigung von Politiken verstanden, weisen Kommissionen tatsächlich diverse Externalisierungs-, Vorverhandlungs-, Vertagungs-, Selbststeuerungs- und symbolische Funktionen auf (Siefken 2019, S. 158 f.). Diese expertokratischen Implikationen gehen

1 Beispiele hierfür sind das vom Bundesministerium für Wirtschaft und Energie seit 2010 durchgeführte jährliche Monitoring zur Erreichung der Ziele des

Energiekonzepts und eine seit 2011 angeschlossene Expertenkommission, welche den Prozess evaluiert. Diverse Gutachten zur Energiewende wurden zuletzt durch den Bundesrechnungshof, die Monopolkommission und den Sachverständigenrat zur Begutachtung der gesamtwirtschaftlichen Entwicklung erstellt. 
von den Kommissionen selbst aus; weitere ergeben sich durch die Rolle, die ihnen im Rahmen der Regierungsarbeit und in demokratischen Verhandlungssystemen eingeräumt wird. Die verstärkte Berufung von Kommissionen und Einholung von Fachgutachten kann sowohl die inhaltliche Strukturierung von Debatten als auch politische Argumentationsmuster beeinflussen. Damit kommt es zu Auswirkungen auf politische Aushandlungsund Entscheidungsfindungsprozesse. In der Energiewende wurden über Expertenkommissionen komplexe Sachverhalte wie der Atom- und Kohleausstieg externalisiert. Vertagungsmuster finden sich insbesondere bei koalitionsinternen Uneinigkeiten etwa bzgl. Ausbaupfaden der erneuerbaren Energien, die durch Arbeitsgruppen-Gründungen adressiert werden.

Anhand der 2018 eingesetzten Kommission für Wachstum, Strukturwandel und Beschäftigung (besser bekannt als Kohlekommission) lassen sich weitere Fallstricke der Delegation von Aushandlungs- und Entscheidungsprozessen an Kommissionen zeigen. So kam es zu Unmut bei lokalpolitischen Akteuren, da die Entwicklung tragfähiger Strukturwandel-Perspektiven für die Regionen gegenüber dem Kohleausstiegsdatum und den Kosten für die Bundesregierung nachrangig erschien. Der durch die demokratischen Legitimationsdimensionen (Input, Throughput und Output) informierte Blick zeigt Grenzen der Kommissionsarbeit auf: Politische Absprachen wurden auch außerhalb getroffen, Bürgerinnen und Bürger wenig beteiligt und nicht umfassend informiert; es lagen Erfahrungs- und Kompetenzasymmetrien unter den Mitgliedern vor und inhaltlich überwogen ökonomische Schwerpunkte (Grothus und Setton 2020). Hinsichtlich des Outputs scheint eine Herausforderung auf, die sich bei stärker partizipativ ausgerichteten TA-Prozessen noch verstärkt: die Übertragung in die Praxis und die Umsetzung oft mühsam errungener Kompromisse sowie detailliert ausgearbeiteter Vorschläge. So ist zwar die Arbeit von Expertenkommissionen von vornherein auf die Genese konkreten Outcomes innerhalb einer festen Zeitspanne ausgerichtet und berücksichtigt die Abgrenzung von Wissens- und Politikregimen. Jedoch kommt es hierdurch zu einer Abkopplung der Entscheidungen, was zu Enttäuschungen und Frustrationserfahrungen aufgrund mangelnden Impacts der Inhalte führt (der in Kontrast zu normativen Implikationen partizipativer Verfahren steht) (Leibenath et al. 2016). Grothus und Setton (2020, S. 298) weisen in diesem Zusammenhang darauf hin, dass nach Abschluss der Kommissionsarbeit der Einfluss zivilgesellschaftlicher Akteure sinkt, während jener von länger und stärker institutionalisierten Akteuren zunimmt (via Lobbying). Demnach ist nicht nur der Arbeitsprozess des beratenden Gremiums (Throughput), sondern auch der Output im Sinne der tatsächlichen Implementierung der Ergebnisse relevant.

Während Steuerungsmodi und Umsetzungsstrategien der Energiewende auf Bundesebene vornehmlich durch Expertengremien geprägt sind, werden Bürgerinnen und Bürger vor Ort durchaus unmittelbar an TA-Prozessen beteiligt. Vermag also die partizipative TA der Energiewende zu größerer demokratischer Legitimation $\mathrm{zu}$ verhelfen?

\section{Partizipative TA - Mehr als Akzeptanz- beschaffung?}

Nach Abels und Bora (2013) handelt es sich bei der partizipativen TA um Instrumente und Methoden,

„[...] die auf eine Beteiligung von Laien und/oder InteressenvertreterInnen (Stakeholder) an TA-Prozessen abzielen und die in unterschiedlicher Weise in Politikberatung eingebunden sind. [...] [D]ie Diskussion zwischen Laien und ExpertInnen [steht] in den meisten Fällen im Vordergrund. [...] Meist sind diese Verfahren deliberativer - erörternder Natur, zum geringen Teil haben sie eine (allerdings schwache) Entscheidungskomponente" (Abels und Bora 2013, S. 109).

Typischerweise steht im Mittelpunkt, dass „ExpertInnen und Laien, Entscheider und Betroffene, GegnerInnen und BefürworterInnen“ darüber verhandeln, ,ob eine umstrittene Technik eingeführt werden soll und wie sie gegebenenfalls zu regulieren ist" (ebd., S. 110). Es lassen sich etwa mit Blick auf Anzahl und Heterogenität der Beteiligten sowie Formen und Funktionen der Beteiligung diverse Verfahren unterscheiden. Viele Modelle folgen dem Ideal der Deliberation (wechselseitige Erörterung und Abwägung) (ebd., S. 118, 121; Kamlage und Reinermann 2020, S. 42).

Die partizipative TA zielt darauf, die demokratische Legitimation von Entscheidungen und Vorhaben zu erhöhen. Konzeptionell lassen sich die verschiedenen Formen von Partizipation und Legitimationsgenerierung in der Energiewende mühelos zu Ansätzen einschlägiger Demokratietheorien in Bezug setzen. Abels und Bora (2013, S. 125) identifizieren drei basale Beteiligungstypen (deliberativ, partizipatorisch, pluralistisch), die in der Praxis zumeist vermischt auftreten. Ebenfalls analytischer Natur ist die Unterscheidung von Renn und Schweizer (2009), die sechs Konzepte von Partizipation umfasst: funktionalistisch (Repräsentation aller Wissensträger), neoliberal (proportionale Repräsentation aller Werte und Zustimmung der betroffenen Öffentlichkeit), deliberativ (wechselseitige Erörterung), anthropologisch (Inklusion neutraler Laien), emanzipatorisch (Emanzipation unterprivilegierter Individuen) und postmodern (Abbildung Pluralität). Im Energiewende-Regime beobachten sie eine Dominanz funktionalistisch-neoliberaler Ausrichtung (Renn und Schweizer 2020, S. 55).

Das bekannte Prozessmodell demokratischer Legitimation nach Easton (1965) ermöglicht es, die Kanäle der Input-, Throughput- und Output-Legitimation zunächst mit Demokratiemodellen und schließlich mit Prozessen und Verfahren in der Energiewende $\mathrm{zu}$ verbinden:

Input-Legitimation: Der im Hinblick auf Anzahl und Meinungsvielfalt möglichst große Einbezug von Personen und Interessen ist im partizipatorischen Demokratiemodell entscheidend (Barber 1984). Partizipative TA tritt im Kontext der Energiewende in deliberativen Formaten wie Planungszellen sowie in emanzipatorischer (z. B. Bottom-up-Initiativen) und funktionalistischer (z. B. von Stakeholdern top-down initiierte Dialog- 
formate) Form auf. Während sowohl aus emanzipatorischer wie auch funktionalistischer Perspektive offene Beteiligungsmöglichkeiten geboten sind, setzen das deliberative und neoliberale Modell auf proportionale Repräsentation von Werten und Präferenzen in der Beteiligtengruppe.

Throughput-Legitimation: Die demokratische Prozessphase bildet die Verarbeitung des demokratischen Inputs ab und wird unterteilt in Willensbildungs-, Implementations- und Evaluierungsphase (Kneip und Merkel 2020, S. 44). Hierbei müssen demokratische Kriterien der Fairness, Neutralität, Offenheit und Transparenz gewährleistet sein (Alcántara et al. 2016, S. 152 ff.). Nach Kneip und Merkel (2020, S. 44) riskiert die Throughput- nungen - sich in lokalen Konflikten durchaus niederschlagen. Da grundsätzliche Dilemmata der Energiewende (z. B. Standortauswahl, Verfahrensgebundenheit) kaum beeinflusst werden können (Leibenath et al. 2016), rückt die Optimierung der existierenden Optionen der Bürgerbeteiligung in den Fokus (Kamlage et al. 2020).

Die beschriebenen Problemlagen lenken den Blick auf etwas, das Schröder (2019) als blinden Fleck der TA beschreibt: Das Politische im Sinne eines tatsächlich ausgetragenen Wettstreits konkurrierender Interessen und Wertvorstellungen nach den Ideen agonistischer Demokratie bleibt unterbelichtet (Mouffe 2013). Dies lassen auch diverse lokale Aushandlungsprozesse

\section{Da grundsätzliche Dilemmata der Energiewende kaum beeinflusst werden können, rückt die Optimierung existierender Optionen der Bürgerbeteiligung in den Fokus.}

Legitimität ,zum ersten Opfer eines exekutivlastigen Dezisionismus“ zu werden, da die Exekutive immer mehr Entscheidungen eigenständig trifft, weshalb der Schaffung indirekter Legitimität (durch andere Kanäle demokratischer Interessenaggregation wie partizipative Formate) eine wachsende Bedeutung $\mathrm{zu}$ kommt. Daraus ergeben sich - auch im Rahmen der TA in der Energiewende - hohe legitimatorische Ansprüche an Gremien und Verfahren, nämlich: dieselben Standards wie in formellinstitutionalisierten Prozessen zu garantieren (die sie schon sui generis kaum erfüllen können).

Output-Legitimation: Die Legitimationskraft von Ergebnissen, ,welche die Bürgerinnen erhoffen und erwarten“, mithin also akzeptieren oder befürworten, ist höher (Kneip und Merkel 2020, S.48). Unpopuläre Ergebnisse können nur zeitweise durch ein ,zufriedenstellendes Niveau“ von Input und Throughput kompensiert werden (ebd.). Dies gelingt in der Energiewende nicht flächendeckend; es kommt zu einem unausgewogenen $\mathrm{Zu}$ stand zwischen Best Practice und Worst Case (Radtke und Renn 2019). Planungsverfahren und gesetzliche Vorgaben lassen kaum Spielräume zu, sodass oft nur wenig Mitbestimmung möglich ist, Expertenmeinungen dominieren und im Falle inputlastiger Verfahren vielstimmige ,Wunschkonzerte“ ins Leere laufen.

Für alle legitimations- bzw. akzeptanzgenerierenden Faktoren in der Energiewende lassen sich Defizite konstatieren. Hildebrand et al. (2017, S. 270) unterscheiden hierbei Repräsentativitäts-, Ressourcen-, (Ergebnis-)Integrations- und Erwartungshaltungsprobleme. Insbesondere bei nicht-formalisierten Verfahren ist die Legitimitätsgenerierung stark einzelfallabhängig (Hitschfeld 2020). Indes bestehen, wie im vorangegangenen Abschnitt beschrieben, bei der Energiewende übergeordnete Steuerungsund Umsetzungsprobleme, die - zumal in Kombination mit der technologisch-ökonomischen Ausrichtung der Energiepolitik sowie der zentralen Bedeutung vorherrschender Expertenmei- im Kontext der Energiewende vermissen, werden doch Differenzen und Kontroversen häufig so weit wie möglich durch Administration und Vorhabenträger top-down eingehegt und oberflächlich befriedet (Cuppen 2018). Dabei steckt viel Konstruktives im Konflikt: So vermochten Bürgerinitiativen das hinter dem Stromnetzausbau stehende Paradigma eines zentralisierten Energiesystems zu dekonstruieren - und setzen sich nun über den Einzelfall hinaus für eine dezentrale Energiewende ein (Neukirch 2017). Einer lokalen Politisierung der Öffentlichkeit(en) wie im Kontext partizipativer TA steht demnach eine durch den verstärkten Einsatz von Expertenkommissionen gekennzeichnete Depolitisierung auf nationaler, speziell parlamentarischer Ebene gegenüber.

\section{Wie weiter mit der Energiewende?}

Vor dem Hintergrund der an Anzahl und Intensität zunehmenden Widerstände gegen ihre lokalen Manifestierungen kommt der gesellschaftlichen Unterstützung der Energiewende zentrale Bedeutung zu. Grundsätzlich vermag TA die Legitimationskraft der Energiewende durch die Berücksichtigung von Expertenmeinungen und Bürgerinteressen zu erhöhen (breite und fundierte Wissensbasis). Dem stehen jedoch im Wesentlichen komplexe Problemlagen entgegen: Erstens eine Dominanz ökonomischtechnologischer Perspektiven aufgrund einschlägig besetzter Expertengremien und fehlender laienbasierter TA, zweitens eine unzureichende Einbettung der Ergebnisse von Expertenkommissionen und Beteiligungsprozessen in politische, insbesondere parlamentarische Entscheidungsprozesse sowie drittens eine sowohl ebenen- und regionen- als auch sektorübergreifend fehlende Verzahnung zwischen expertendominierter und partizipativer TA. 
In jüngerer Zeit lässt sich beobachten, dass die Energiewende zunehmend in populistische Narrative eingespeist wird (Radtke et al. 2019); ein möglicher Zusammenhang zwischen Technokratie und Populismus wird häufig diskutiert (Landwehr 2018). Dazu passt die Dominanz top-down-geprägter Verfahren der Öffentlichkeitsbeteiligung (in deren Rahmen auch mehr oder minder partizipative TA stattfindet), die wenig tatsächliche Mitbestimmung erlauben (Huge und Roßnagel 2018; Leibenath et al. 2016). Bislang existieren kaum Verschränkungen zwischen lokalen Anliegen bzw. Verfahren und nationaler Politikgestaltung, da zwischen beiden Sphären eine systemisch-strukturell bedingte Kluft besteht, welche die Rückbindung, von unten nach nach stärker Berücksichtigung finden. Dabei würden zwar nicht die Abkehr von fossilen Energieträgern und Atomkraft zur Disposition stehen, wohl aber wesentliche Pfeiler der Ausgestaltung des zukünftigen Energiesystems, die parlamentarisch sehr viel stärker verhandelt werden könnten - bestenfalls konsultativ durch Laien, etwa Bürgerräte, gestützt.

Verfahren der partizipativen TA sollten nur mit dem Impetus eines genuinen Interesses an der Mitgestaltung durch Bürgerinnen und Bürger lanciert, Konflikte nicht leichthin befriedet werden. Die Steuerung und Umsetzung der Energiewende würden von der stärkeren Einbindung und Wertschätzung von Laienperspektiven wesentlich profitieren, während umgekehrt

\section{Impulse für eine Neu-Ausrichtung und Akzentuierung einer Energiewende-Politik 2.0 müssten bald gesetzt werden.}

oben' erschwert. Für die Vermittlung bietet sich eine Stärkung vielfach bereits vorhandener, intermediärer Institutionen an, die nicht nur (wie bisher) zur Vermittlung von Energie(wende)politiken, sondern auch zur Erhöhung politischer Responsivität durch Einspeisen spezifischer, teils stärker regionalisierter Interessen in den Policy-Prozess herangezogen werden könnten. Beispielhaft lassen sich die Energieagenturen auf Länderebene anführen. Eine stärkere Rückkopplung ist indes nicht nur zwischen den föderalen Ebenen erforderlich, auch bestehende Entscheidungsfindungsprozesse innerhalb politischer Gremien und Verfahren können durch zusätzliche, gezielt eingesetzte partizipative Formate inhaltlich angereichert werden und an Legitimationskraft gewinnen (Brettschneider 2020; Radtke und Saßmannhausen 2020). Mit Blick auf die Energiewende bietet sich eine stärkere Betonung funktionalistisch-deliberativer Ansätze (Renn und Schweizer 2020, S. 55) sowie die kontextsensible Kombination verschiedener Modelle an, um ,deren jeweilige Potenziale zur Umsetzung der Energiewende auszuschöpfen und dabei die Limitierungen möglichst zu vermeiden“ (Schweizer 2015, S. 29).

Zuletzt liefert die Literatur zur TA einen wertvollen Ansatzpunkt für die Zukunft der Energiewende: die „Ergebnisoffenheit" nämlich, welche die Pole „Akzeptanzbeschaffung“ und „Technikveränderung“ verbindet (Grunwald 2010, S. 288). Wenngleich physikalische Gesetze und technische Erfordernisse zweifellos bestehen, so ist doch das zukünftige EnergiesystemDesign, wie es aktuell ins Auge gefasst wird, keineswegs alternativlos. Lange bevor sie allerorten zu Unmut und Protesten sowie langwierigen Klageverfahren führte, galt die Dezentralität der erneuerbaren Energien als Ermöglichungsfaktor für eine demokratischere, d. h. bürgerorientierte Energieversorgung, was unterdessen nicht an Geltungskraft eingebüßt hat. Die Perspektiven und Präferenzen der Bürgerinnen und Bürger sollten dem- eine stärker reflexive Vermittlung, nach unten“ ein ungekanntes Maß an Diffusion von Einstellungen, Wissen und Expertise sowie eine Robustheit getroffener Entscheidungen bieten könnte, was der Legitimationskraft sehr zuträglich wäre (Natarajan 2017). Impulse für eine Neu-Ausrichtung und Akzentuierung einer Energiewende-Politik 2.0, die stärker auf Rückkopplung und Intermediäre setzt, Parlamente mehr einbezieht und hierfür Gestaltungs- und Handlungsspielräume zur Schaffung einer eigenständigen diskursiven Arena einräumt, müssten mit Blick auf den zeitlichen Korridor bis 2050 freilich bald gesetzt werden.

\section{Literatur}

Abels, Gabriele; Bora, Alfons (2013): Partizipative Technikfolgenabschätzung und -bewertung. In: Georg Simonis (Hg.): Konzepte und Verfahren der Technikfolgenabschätzung. Wiesbaden: Springer Fachmedien, S. 109-128. DOI: 10.1007/978-3-658-02035-4_7

AEE - Agentur für Erneuerbare Energien (2020): Akzeptanz. Online verfügbar unter https://www.unendlich-viel-energie.de/themen/akzeptanzerneuerbarer/akzeptanz-umfrage, zuletzt geprüft am 03.10.2020.

Alcántara, Sophia; Bach, Nicolas; Kuhn, Rainer; Ullrich, Peter (Hg.) (2016): Demokratietheorie und Partizipationspraxis. Analyse und Anwendungspotentiale deliberativer Verfahren. Wiesbaden: Springer VS. DOI: 10.1007/ 978-3-658-11221-9.

Barber, Benjamin (1984): Strong democracy. Participatory politics for a new age. Berkeley: University of California Press.

Brettschneider, Frank (Hg.) (2020): Bau- und Infrastrukturprojekte. Dialogorientierte Kommunikation als Erfolgsfaktor. Wiesbaden: Springer VS. DOI: 10.1007/978-3-658-28235-6.

Chemnitz, Christine (2019): Die Umsetzung der Energiewende im Föderalismus. Koordination und Steuerung durch mehr Politikverflechtung. In: dms der moderne staat - Zeitschrift für Public Policy, Recht und Management 12 (1), S. 116-144. DOI: 10.3224/dms.v12i1.06. 
Cuppen, Eefje (2018): The value of social conflicts. Critiquing invited participation in energy projects. In: Energy Research \& Social Science 38, S. 28-32. DOI: 10.1016/j.erss.2018.01.016.

Czada, Roland (2019): Politikwenden und transformative Politik in Deutschland. In: dms - der moderne staat 12 (2), S. 400-417. D0I: 10.3224/dms.v12i2.12.

Die Bundesregierung (2002): Perspektiven für Deutschland. Unsere Strategie für eine nachhaltige Entwicklung. Online verfügbar unter https:// www.bundesregierung.de/resource/blob/997532/464596/a9a177234880a22 8ae90b08106175dce/perspektiven-fuer-deutschland-langfassung-data.pdf, zuletzt geprüft am 27.10.2020.

Easton, David (1965): A systems analysis of political life. New York: Wiley.

Ethik-Kommission Sichere Energieversorgung (2011): Deutschlands Energiewende. Ein Gemeinschaftswerk für die Zukunft. Berlin: Die Bundesregierung. Online verfügbar unter https://archiv.bundesregierung.de/resource/ blob/656922/394384/962baf09452793c8a87402c9ee347379/2011-07-28abschlussbericht-ethikkommission-data.pdf, zuletzt geprüft am 27. 10.2020.

Gleich, Arnim von (2013): Prospektive Technikbewertung und Technikgestaltung zur Umsetzung des Vorsorgeprinzips. In: Georg Simonis (Hg.): Konzepte und Verfahren der Technikfolgenabschätzung. Wiesbaden: Springer Fachmedien, S. 51-73. DOI: 10.1007/978-3-658-02035-4_4.

Grothus, Antje; Setton, Daniela (2020): Die „Kohlekommission“ aus zivilgesellschaftlicher Perspektive. Chancen und Herausforderungen bei der Partizipation in Expertengremien. In: Forschungsjournal Soziale Bewegungen, 33 (1), S. 282-304. DOI: 10.1515/fjsb-2020-0023.

Grunwald, Armin (2010): Technikfolgenabschätzung. Eine Einführung. Berlin: edition sigma.

Gründinger, Wolfgang (2017): Drivers of energy transition. How interest groups influenced energy politics in Germany. Wiesbaden: Springer VS. DOI: 10.1007/978-3-658-17691-4.

Haas, Tobias (2017): Die politische Ökonomie der Energiewende. Deutschland und Spanien im Kontext multipler Krisendynamiken in Europa. Wiesbaden: Springer VS. DOI: 10.1007/978-3-658-17319-7.

Habermas, Jürgen (2013): Im Sog der Technokratie. Kleine politische Schriften XII. Berlin: Suhrkamp.

Haring, Sophie (2010): Herrschaft der Experten oder Herrschaft des Sachzwangs? Technokratie als politikwissenschaftliches »ProblemEnsemble«. In: Zeitschrift für Politik 57 (3), S. 243-264.

Hildebrand, Jan; Rau, Irina; Schweizer-Ries, Petra (2017): Höhere öffentliche Akzeptanz durch bessere Beteiligungsverfahren? In: UVP-Report 31 (4), S. 269-273.

Hitschfeld, Uwe (2020): Steuerung von Partizipation und Anforderungen an das Verfahrensdesign. In: Astrid Lorenz, Christian Pieter Hoffmann und Uwe Hitschfeld (Hg.): Partizipation für alle und alles? Fallstricke, Grenzen und Möglichkeiten. Wiesbaden: Springer VS, S. 345-362. D0I: 10.1007/ 978-3-658-27898-4_19.

Huge, Antonia; Roßnagel, Alexander (2018): Möglichkeiten der Öffentlichkeitsbeteiligung in Planungs- und Genehmigungsverfahren von Windenergieanlagen. In: Lars Holstenkamp und Jörg Radtke (Hg.): Handbuch Energiewende und Partizipation. Wiesbaden: Springer VS, S. 613-625. DOI: 10.1007/978-3-658-09416-4_37.

Kamlage, Jan-Hendrik; Drewing, Emily; Reinermann, Julia; de Vries, Nicole; Flores, Marissa (2020): Fighting fruitfully? Participation and conflict in the context of electricity grid extension in Germany. In: Utilities Policy 64. DOI: 10.1016/j.jup.2020.101022.
Kamlage, Jan-Hendrik; Reinermann, Julia (2020): Die doppelte Normativität der partizipativen Technikfolgenabschätzung. Entwicklungen, Funktionen und Herausforderungen. In: Linda Nierling und Helge Torgersen (Hg.): Die neutrale Normativität der Technikfolgenabschätzung. Konzeptionelle Auseinandersetzung und praktischer Umgang. Baden-Baden: Nomos, S. 39-56. DOI: 10.5771/9783748907275-39.

Kneip, Sascha; Merkel, Wolfgang (2020): Demokratische Legitimität. Ein theoretisches Konzept in empirisch-analytischer Absicht. In: Sascha Kneip, Wolfgang Merkel und Bernhard Weßels (Hg.): Legitimitätsprobleme. Zur Lage der Demokratie in Deutschland. Wiesbaden: Springer VS, S. 25-55. DOI: 10.1007/978-3-658-29558-5_2.

Landwehr, Claudia (2018): Technokratie und Populismus - Entstellungen der Demokratie? Demokratietheorie und demokratische Praxis. In: Winfried Thaa und Christian Volk (Hg.): Formwandel der Demokratie. Baden-Baden: Nomos, S. 155-174.

Leibenath, Markus; Wirth, Peter; Lintz, Gerd (2016): Just a talking shop? Informal participatory spatial planning for implementing state wind energy targets in Germany. In: Utilities Policy 41, S. 206-213.

Mouffe, Chantal (2013): Agonistics. Thinking the world politically. London: Verso.

Natarajan, Lucy (2017): Socio-spatial learning. A case study of community knowledge in participatory spatial planning. In: Progress in Planning 111, S.1-23, DOI: 10.1016/j.progress.2015.06.002.

Neukirch, Mario (2017): Die Dynamik des Konflikts um den Stromtrassenbau. Stabilität, Wandel oder Stagnation? SOI Discussion Paper 2017-04. Stuttgart: Universität Stuttgart. Online verfügbar unter https://www.sowi.uni-stuttgart. de/dokumente/forschung/soi/soi_2017_4_Neukirch.Dynamik.Konflikt. Stromtrassenbau.pdf, zuletzt geprüft am 27.10.2020.

Neunecker, Martina (2016): Partizipation trifft Repräsentation. Die Wirkungen konsultativer Bürgerbeteiligung auf politische Entscheidungen. Wiesbaden: Springer VS. DOI: 10.1007/978-3-658-13071-8.

Radtke, Jörg (2018): Energie. In: Rüdiger Voigt (Hg.): Handbuch Staat. Wiesbaden: Springer Fachmedien, S. 1411-1421. DOI: 10.1007/978-3-658-20744-1_127.

Radtke, Jörg (2020): Das Jahrhundertprojekt der Nachhaltigkeit am Scheideweg. Wie kann die Energiewende in Deutschland breite gesellschaftliche Unterstützung finden? In: Zeitschrift für Politikwissenschaft 30 (1), S. 97-111. DOI: 10.1007/s41358-020-00215-6.

Radtke, Jörg; Canzler, Weert; Schreurs, Miranda; Wurster, Stefan (Hg.) (2019): Energiewende in Zeiten des Populismus. Wiesbaden: Springer VS. DOI: 10.1007/978-3-658-26103-0.

Radtke, Jörg; Renn, Ortwin (2019): Partizipation und bürgerschaftliches Engagement in der Energiewende. In: Weert Canzler und Jörg Radtke (Hg.): Energiewende. Eine sozialwissenschaftliche Einführung. Wiesbaden: Springer VS, S. 283-316. DOI: 10.1007/978-3-658-26327-0_10.

Radtke, Jörg; Saßmannhausen, Sheree (2020): Auf dem Weg zur responsiven Demokratie? Online-Öffentlichkeitsbeteiligung in der Stadtentwicklung als aktiver Link zwischen Kommunalpolitik und Bevölkerung. In: Zeitschrift für Politikwissenschaft 30 (2), S. 329-358. D0I: 10.1007/s41358-020-00233-4.

Renn, Ortwin; Schweizer, Pia-Johanna (2009): Inclusive risk governance. Concepts and application to environmental policy making. In: Environmental Policy and Governance 19 (3), S. 174-185. DOI: 10.1002/eet.507.

Renn, Ortwin; Schweizer, Pia-Johanna (2020): Inclusive governance for energy policy making. Conceptual foundations, applications, and lessons learned. In: Ortwin Renn, Frank Ulmer und Anna Deckert (Hg.): The role 
of public participation in energy transitions. San Diego: Academic Press, S. 39-79.

Rothe, Kerstin (2017): Pragmatismus in politischen Entscheidungsprozessen. Baden-Baden: Nomos. DOI: 10.5771/9783845281445.

SRU - Sachverständigenrat für Umweltfragen (2011): Wege zur $100 \%$ erneuerbaren Stromversorgung. Sondergutachten. Berlin: Erich Schmidt Verlag. Schröder, Julia (2019): Das Politische in der Technikfolgenabschätzung. Reflexionen mit der pluralen, radikalen Demokratietheorie von Laclau und Mouffe. In: TATuP - Zeitschrift für Technikfolgenabschätzung in Theorie und Praxis 28 (3), S. 62-67. D0I: 10.14512/tatup.28.3.62.

Schweizer, Pia-Johanna (2015): Partizipation bei der Energiewende und beim Ausbau der Stromnetze. Philosophische Fundierung. TAB-Brief Nr. 45. Berlin: Büro für Technikfolgen-Abschätzung beim Deutschen Bundestag.

Setton, Daniela (2019): Soziales Nachhaltigkeitsbarometer der Energiewende 2018. Potsdam: Institute for Advanced Sustainability Studies (IASS).

Siefken, Sven (2019): Expertenkommissionen der Bundesregierung. In: Svenja

Falk, Manuela Glaab, Andrea Römmele, Henrik Schober und Martin Thunert (Hg.): Handbuch Politikberatung. Wiesbaden: Springer VS, S. 145-161. DOI: 10.1007/978-3-658-03483-2_14

TAB - Büro für Technikfolgenabschätzung beim Deutschen Bundestag (2020): Laufende Untersuchungen. Online verfügbar unter https://www.tab-beimbundestag.de/de/untersuchungen/laufende-untersuchungen.html, zuletzt geprüft am 03.10.2020.

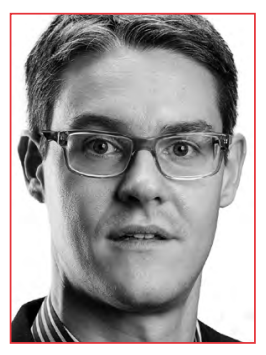

\section{DR.JÖRG RADTKE}

ilst Politikwissenschaftler, Leiter des Projekts "Creactive Citizen" und Ko-Leiter des Projekts "Eine neue Konfliktkultur für die Energiewende“ am Seminar für Sozialwissenschaften, Universität Siegen. Er promovierte 2015 zum Thema Bürgerenergie. Er ist Sprecher der Themengruppe Energietransformation der DVPW sowie des Forschungsnetzwerkes Energiegenossenschaften.

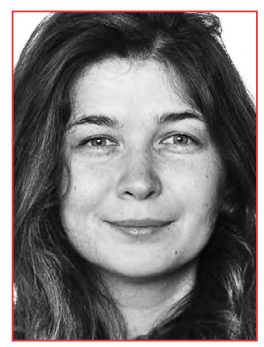

\section{EMILY DREWING}

ist Sozialwissenschaftlerin und beschäftigt sich als wissenschaftliche Mitarbeiterin an der Universität Siegen (Seminar für Sozialwissenschaften) und an der Ruhr-Universität Bochum (Geographisches Institut) mit Partizipation und Konflikten im Kontext der Energiewende vor dem Hintergrund zeitgenössischer Megatrends. Sie promoviert über Energiegenossenschaften.

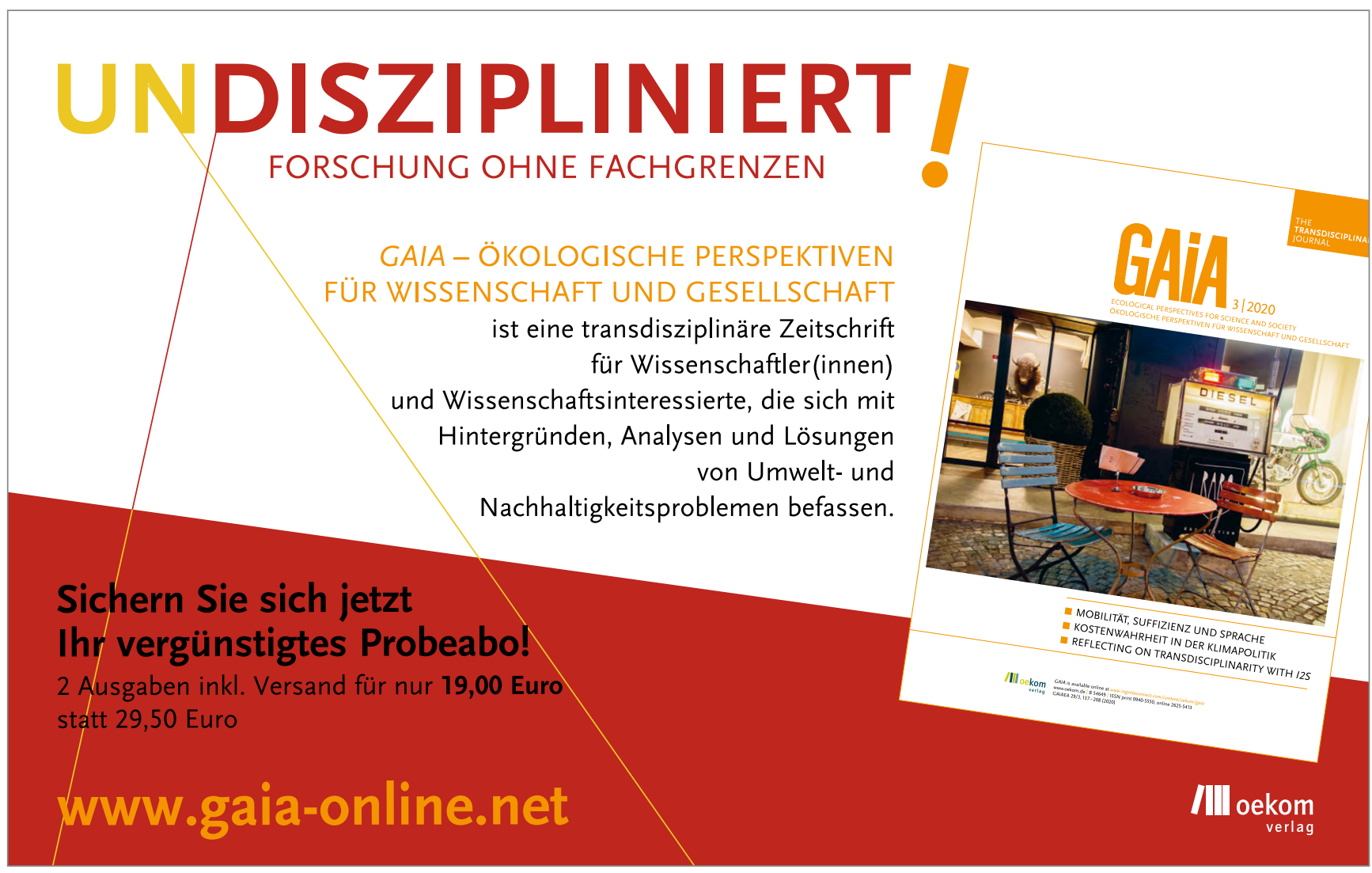

